\title{
Namlu Stabilizasyonunun Kayan Kipli Kontrol Yöntemi Desteği İle Dört Bacakı Bir Robot Üzerinde Uygulanması
}

\author{
Ahmet Burak TATAR*, Oğuz YAKUT \\ Firat Üniversitesi, Mekatronik Mühendisliği, Elazı̆̆ \\ (ORCID: 0000-0001-5848-443X) (ORCID: 0000-0002-0986-1435)
}

\begin{abstract}
Öz
Savunma alanında yapılan çalışmalarda ele alınan silah sistemlerinin kolay kontrol edilebilir ve yüksek hassasiyetli olması beklenmektedir. Silah sistemlerinin başarılı bir şekilde hedef takibi yapabilmesi namlu stabilizasyon sistemi ile mümkündür. Silah kulesi stabilizasyon çalışmaları ile geliştirilen savunma teknolojilerinin önemi günümüzde gittikçe artmaktadır. Robot teknolojisinin gelişmesine bağlı olarak gelecekte savunma sistemlerinde robotların kullanımı da kaçınılmaz olacaktır. Bu çalışmada 4 ayaklı bir robot üzerine yerleştirilmiş namlu sisteminin stabilizasyonu ele alınmıştır. Bunun için, üzerine namlu sistemi yerleştirilmiş 4 bacaklı bir robotun düzlemsel hareketini temsil eden bir matematiksel model elde edilmiştir. Sistem toplamda 12 bağımsız eksenden oluşmaktadır. Kayan kipli kontrol yöntemi kullanılarak robotun hareketli durumlarında namlunun stabilizasyonu için kontroller gerçekleştirilmiştir. Bununla birlikte, robotun yürüme hareketinin kontrolünde PID kontrol yönteminden yararlanılmıştır. Bu çalışma ile, üzerine silah kulesi yerleştirilmiş dört bacaklı bir robotun, savunma sanayisinde kullanılabileceği ve namlu stabilizasyonunun kayan kipli kontrol ile gerçekleştirilebileceği ortaya konulmuştur. Simülasyonlar için MATLAB paket programı kullanılmış ve elde edilen sonuçlar grafiksel olarak değerlendirilmiştir.
\end{abstract}

Anahtar Kelimeler: Dört Bacaklı Robot, Namlu Stabilizasyonu, Kayan Kipli Kontrol, Dinamik Model.

\section{Application of the Gun Barrel Stabilization on a Four-Legged Robot with the aid of Sliding Mode Control Method}

\begin{abstract}
It is expected that the weapon systems discussed in the field of defense will be easily controllable and high sensitivity. It is possible with the gun barrel stabilization system that the weapon systems can successfully follow the target. The importance of defense technologies developed with gun barrel stabilization studies is increasing day by day. Due to the development of robot technology, the usage of robots in defense systems in the future will also be inevitable. In this study, stabilization of the gun barrel system placed on a 4-legged robot is discussed. For this, a mathematical model representing the planar motion of a 4-legged robot with a gun barrel system was obtained. The system consists of 12 independent axes in total. Controls were realized for the gun barrel stabilization in moving situations by using the sliding mode control method. In this study, it was revealed that a four-legged robot with a gun turret placed on it can be used in the defense industry and the gun barrel stabilization can be implemented with sliding-mode control. MATLAB package program was used for the simulations and the results were analyzed graphically.
\end{abstract}

Keywords: Four-legged robot, Gun Barrel Stabilization, Sliding Mode Control, Dynamic Model.

*Sorumlu yazar: atatar@firat.edu.tr

Geliş Tarihi:15.03.2019, Kabul Tarihi: 09.08.2019 


\section{Giriş}

Ülkeler daima, bir birine karşı üstünlük sağlamak ve tehditlere karşı caydırıcılı̆̆ı arttırmak amacıyla güçlü, etkili ve gelişmiş silah sistemlerine sahip olma arzusundadır. Ulusların bu alandaki çalışmaları günümüzde de artan bir hızla devam etmektedir [1].

Günümüz savunma sanayi teknolojisi ile geliştirilen yeni nesil silah sistemlerinin temel amacı, her türlü bozucu etki karşısında belirlenen hedefin başarılı bir şekilde vurulmasını sağlamaktır. Aynı zamanda, yaşadığımız dönem içerisindeki harp koşullarında, daha hareketli ve daha hızlı tepki verebilen silah sistemleri tercih edilmektedir. Günümüzün namlu sistemlerinin bir diğer özelliği, hareketli hedefler için yüksek atış doğruluğu sağlayan otomatik hedef izlemedir [2]. Bu silah sistemleri içerisinde, hareketli araç üzerinden yapılan atışın başarılı olması namlu stabilizasyonu ile mümkündür. Namlu stabilizasyon sistemi sadece bir hedef takip sistemi değil ayn zamanda namlunun hedef bölgesinde kalmasını sağlayan arazi şartlarından en az etkilenen yüksek vuruş yüzdesine sahip atışlar yapılmasına yardımcı olan bir sistemdir[3]. Yapılan çalışmalarda daha önce bahsedildiği üzere bu sistemlerde edinilmesi gerekli olan en önemli ve en kritik yeteneklerden biri silah kulesi sistemlerinin "hareket halindeki ateş" yeteneğidir. Bu kabiliyet temel olarak namlu stabilizasyonu ile elde edilmektedir. Bu yetenek, silah üzerindeki yönünü, engebeli bir arazide hareket eden araç tarafindan oluşturulan rastgele bozucu etkiler altında bile zemindeki referansa göre sabit tutması anlamına gelmektedir [4]. Son y1llarda namlu stabilizasyon sistemleri üzerine bazı çalışmalar yapılmıştır. Korobiichuk, silah sistemlerinin stabilizasyonunda kullanılan bir sensor çeşidinin matematiksel modeli üzerine çalışmıştır [5]. Kline ve arkadaşları, silah ile hedef belirleme problemleri üzerine çalışma yapmıştır [6].

Dünyada savunma sanayisine katkılar sağlayan robot teknolojisinin eklem bacaklı robotlar alanında, çok önemli ilerlemeler sağlanmıştır. Bacaklı robotlar, tekerlekli mobil robotlara göre zorlu arazi koşullarında manevra kabiliyeti açısından daha çok tercih edilmektedir[7]. Dört bacaklı robotlar diğer bacaklı robotlara göre daha kararlı ve daha çok yük taşıma kapasitesine sahiptir [8]. Ayrıca Gao ve ekibi, bacak mekanizmasının geniş çalışma alanına ve yüksek serbestlik derecesine sahip olduğunu ve hareket sürecindeki pozisyon değişikliği konusunda gerçek zamanlı geri bildirim sağladığını belirtmişlerdir [9]. Bu çalışmada 4 bacaklı bir robotun düzlemsel dinamik modeli elde edilerek robotun yürüyüş kontrol simülasyonları gerçekleştirilmiştir. Namlu stabilizasyonuna yönelik çalışmalar için robotun gövdesi üzerine hareketli bir namlu sistemi eklenmiştir. Yürüyen veya sıçrayan bir robotun gövde hareketleri namlu sistemi için bozucu etkiye sebep olmaktadır. Kayan kipli kontrol yöntemi, yüksek hızlı ve doğrusal olmayan bir geri beslemeye sahip kontrol yöntemidir. Özellikle parametrik belirsizliğe ve bozucu etkilere karşı dayanıklı bir kontrol cevabı sunmaktadır. Bu çalışmada, robot üzerinde bozucu etkilere maruz kalan namlunun arzu edilen konumda tutulabilmesi için namluya kayan kipli kontrol yöntemi uygulanmıştır. Simülasyon sonuçları grafiksel olarak sunulmuş ve animasyonlarla desteklenmiştir. Böylece bu çalışma ile, dört bacaklı bir robot üzerine yerleştirilen bir namlu sisteminin, stabilizasyonunun gerçeklenebilmesinde kayan kipli kontrol yönteminin başarısı ortaya konulmuştur. Simülasyonlar, Matlab paket programında bulunan Runge-Kutta sayısal yaklaşım metodu kullanılarak gerçekleştirilmiştir.

\section{Dört Bacakı Robotun Matematiksel Modeli}

\subsection{Fiziksel model}

Düzlemsel 4 bacaklı robotun fiziksel modeli Şekil 1'de gösterilmiştir. Robotun düzlemsel hareketi göz önünde bulundurularak matematiksel modeli elde edilmiştir. Elde edilen bu modele robotun 4 bacağı da eklenmiştir. Dolayısıyla robotun trot, gallop ve pace gibi yürüyüş tipleri modele uygulanabilmektedir. 


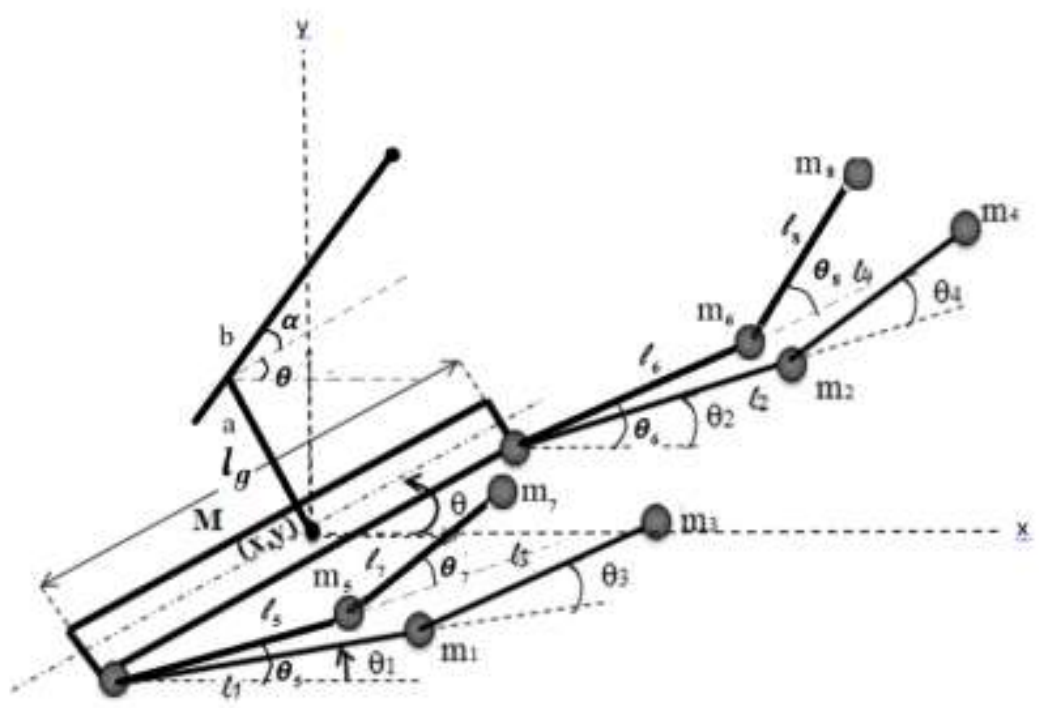

Şekil 1. Dört Bacaklı Robotun Fiziksel Modeli

\subsection{Hareket denklemleri}

Robotun fiziksel modelinde, her bir bacağın 2 serbestlik derecesine ve döner eklemlere sahip olduğu kabul edilmiştir. Robot gövdesi yatayda $\mathrm{x}$-ekseni ve düşeyde y-ekseni doğrultusunda öteleme hareketi yapabilirken merkezde düzleme dik eksen etrafinda dönme hareketi yapabilmektedir. Robot gövdesinin orta merkezinden a mesafesi kadar yukarısına namlu sistemi yerleştirilmiştir. Namlu uzunluğu $b$ boyundadır ve bir döner mafsal yardımıyla hareket düzlemine dik eksen etrafında, robotun gövdesine göre $\alpha$ açısal dönme hareketini gerçekleştirebilmektedir.

Bu fiziksel model yardımıyla robotun hareket denklemleri matematiksel olarak elde edilmiştir. Bunun için Lagrange-Euler yöntemi kullanılmıştır. Lagrange-Euler yöntemi, belirlenen bir sistemin kinetik ve potansiyel enerjilerini dikkate alarak hareket denklemlerini sistematik olarak türetmek için kullanılmaktadır [10]. Bununla birlikte Beckers ve ekibi, Euler-Lagrange(EL) sistemlerinin, hareket denklemi elde etmek için çok önemli ve büyük bir dinamik sistem sınıfı olduğunu ifade etmektedirler [11]. Bu yöntemde, sistemin hareketli elemanlarının hareket denklemlerini elde etmek için kullanılan temel denklem (1)'de verilmiştir.

$$
\frac{d}{d t} \frac{\partial L}{\partial \dot{q}_{l}}\left(q, \dot{q}_{l}\right)-\frac{\partial L}{\partial q_{i}}\left(q, \dot{q}_{l}\right)=\tau_{i} \quad 1 \leq i \leq n
$$

Burada $n$ serbestlik derecesini, $q_{i}$ sistemdeki hareketli elemanın sahip olduğu konumu, $i$ hareketli eleman indisini belirtmektedir. Lagrange $(L)$ denklemi, sistemin toplam kinetik enerji ifadesinden $(T)$, sistemin toplam potansiyel enerji ifadesinin $(V)$ farkının alınması ile elde edilmektedir. Dolayısıyla, sistemin Lagrange ifadesi ve (2), (3) ve (4)’teki gibi elde edilmiştir.

$$
\begin{aligned}
L= & T-V \\
T= & \frac{1}{2} M\left(\dot{x}^{2}+\dot{y}^{2}\right)+\frac{1}{2} I \dot{\theta}^{2}+\frac{1}{2} m_{1}\left(\dot{x}_{1}^{2}+\dot{y}_{1}^{2}\right)+\frac{1}{2} m_{2}\left(\dot{x}_{2}^{2}+\dot{y}_{2}^{2}\right)+\frac{1}{2} m_{3}\left(\dot{x}_{3}^{2}+\dot{y}_{3}^{2}\right)+ \\
& \frac{1}{2} m_{4}\left(\dot{x}_{4}^{2}+\dot{y}_{4}^{2}\right)+\frac{1}{2} m_{5}\left(\dot{x}_{5}^{2}+\dot{y}_{5}^{2}\right)+\frac{1}{2} m_{6}\left(\dot{x}_{6}^{2}+\dot{y}_{6}^{2}\right)+\frac{1}{2} m_{7}\left(\dot{x}_{7}^{2}+\dot{y}_{7}^{2}\right)+\frac{1}{2} m_{8}\left(\dot{x}_{8}^{2}+\dot{y}_{8}^{2}\right) \\
& +\frac{1}{2} m_{n}\left(\dot{x}_{n}^{2}+\dot{y}_{n}^{2}\right) \\
V= & M g y+m_{1} g y_{1}+m_{2} g y_{2}+m_{3} g y_{3}+m_{4} g y_{4}+m_{5} g y_{5}+m_{6} g y_{6}+m_{7} g y_{7}+m_{8} g y_{8} \\
& +m_{n} g y_{n}
\end{aligned}
$$
belirtilmiştir;

Kinetik ve potansiyel enerji ifadelerinde görülen fiziksel parametreler Tablo 1'de işlevleriyle 
Tablo 1. Robotun Fiziksel Parametreleri

\begin{tabular}{clcl}
\hline Sembol & \multicolumn{1}{c}{ Açıklama } & Sembol & \multicolumn{1}{c}{ Açıklama } \\
\hline$M$ & Robot Gövdesinin Kütlesi & $\theta_{1-8}$ & Bacak Uzuvlarının Açısal \\
$x$ & Robot Gövdesinin Yatay & & Konumları \\
& Eksendeki Konumu & $l_{g}$ & Robot Gövdesinin \\
$y$ & Robot Gövdesinin Yatay & & Bacak Uzuvlarının \\
& Eksendeki Konumu & $l_{1-8}$ & Uzunlukları \\
$m_{1-8}$ & Robot Bacak Uzuvlarının & & Kule Uzunluğu \\
& Kütleleri & $\mathrm{a}$ & Namlu Uzunluğu \\
$x_{1-8}$ & Robot Bacak Uzuvlarının & $\mathrm{b}$ & Namlunun Gövdeye Göre \\
& Yatay Eksendeki Konumları & $\alpha$ & Bağıl Açısal Konumu \\
$y_{1-8}$ & Robot Bacak Uzuvlarının & & Namlunun Yatay \\
& Düşey Eksendeki Konumları & $x_{n}$ & Eksendeki Konumu \\
I & Robot Gövdesinin Atalet & & Namlunun Düşey \\
& Momenti & $y_{n}$ & Eksendeki Konumu \\
$\theta$ & Robot Gövdesinin Düzleme & & Namlunun Kütlesi \\
& Dik Eksene Göre Açısal & $m_{n}$ & \\
& Konumu & &
\end{tabular}

Lagrange-Euler yönteminde hareket denklemlerini elde etmek için öncelikle gövdenin ve tüm bacakların (5)-(22)'de verildiği gibi konum ifadelerinin belirlenmesi gerekir.

$$
\begin{aligned}
& x_{1}=x-\frac{l_{g}}{2} \cos \theta+l_{1} \cos \theta_{1} \\
& y_{1}=y-\frac{l_{g}}{2} \sin \theta+l_{1} \sin \theta_{1} \\
& x_{2}=x+\frac{l_{g}}{2} \cos \theta+l_{2} \cos \theta_{2} \\
& y_{2}=y+\frac{l_{g}}{2} \sin \theta+l_{2} \sin \theta_{2} \\
& x_{3}=x-\frac{l_{g}}{2} \cos \theta+l_{1} \cos \theta_{1}+l_{3} \cos \left(\theta_{1}+\theta_{3}\right) \\
& y_{3}=y-\frac{l_{g}}{2} \sin \theta+l_{1} \sin \theta_{1}+l_{3} \sin \left(\theta_{1}+\theta_{3}\right) \\
& x_{4}=x+\frac{l_{g}}{2} \cos \theta+l_{2} \cos \theta_{2}+l_{4} \cos \left(\theta_{2}+\theta_{4}\right) \\
& y_{4}=y+\frac{l_{g}}{2} \sin \theta+l_{2} \sin \theta_{2}+l_{4} \sin \left(\theta_{2}+\theta_{4}\right) \\
& x_{5}=x-\frac{l_{g}}{2} \cos \theta+l_{5} \cos \theta_{5} \\
& y_{5}=y-\frac{l_{g}}{2} \sin \theta+l_{5} \sin \theta_{5} \\
& x_{6}=x+\frac{l_{g}}{2} \cos \theta+l_{6} \cos \theta_{6} \\
& y_{6}=y+\frac{l_{g}}{2} \sin \theta+l_{6} \sin \theta_{6} \\
& x_{7}=x-\frac{l_{g}}{2} \cos \theta+l_{5} \cos \theta_{5}+l_{7} \cos \left(\theta_{5}+\theta_{7}\right) \\
& y_{7}=y-\frac{l_{g}}{2} \sin \theta+l_{5} \sin \theta_{5}+l_{7} \sin \left(\theta_{5}+\theta_{7}\right) \\
& x_{8}=x+\frac{l_{g}}{2} \cos \theta+l_{6} \cos \theta_{6}+l_{8} \cos \left(\theta_{6}+\theta_{8}\right) \\
& y_{8}=y+\frac{l_{g}}{2} \sin \theta+l_{6} \sin \theta_{6}+l_{8} \sin \left(\theta_{6}+\theta_{8}\right) \\
& x_{n}=x-a \sin \theta+b \cos (\alpha+\theta) \\
& y_{n}=y+a \cos \theta+b \sin (\alpha+\theta)
\end{aligned}
$$

$\mathrm{Bu}$ konum ifadelerinin zamana göre türevleri kinetik enerji için gerekli hız ifadelerini vermektedir. Bu hız denklemlerinin de kullanımı ile genel Lagrange denklemi Ek-1'deki gibidir. Bununla birlikte, robotun hareketlerini gerçekleştirebilmesi için gerekli olan uygun tork değerlerinin matematiksel ifadesi de Ek-2'de verilmiştir. 
Hareketli robotun bacaklarının yer ile teması sonucunda oluşan zemin tepki kuvvetlerinin robot gövdesine olan tesirlerinin hesaplanabilmesi için Jakobiyen matrislerine ihtiyaç duyulmaktadır. Ele alınan düzlemsel hareket için zemin tepki kuvvetlerinin sadece $\mathrm{x}$ ve y bileşenleri göz önünde bulundurulmuş ve gerekli jakobiyen matrisi (23) ifadesi kullanılarak elde edilmiştir.

$[J]=\left[\begin{array}{lllllllllll}\frac{\partial x_{i}}{\partial x} & \frac{\partial x_{i}}{\partial y} & \frac{\partial x_{i}}{\partial \theta} & \frac{\partial x_{i}}{\partial \theta_{1}} & \frac{\partial x_{i}}{\partial \theta_{2}} & \frac{\partial x_{i}}{\partial \theta_{3}} & \frac{\partial x_{i}}{\partial \theta_{4}} & \frac{\partial x_{i}}{\partial \theta_{5}} & \frac{\partial x_{i}}{\partial \theta_{6}} & \frac{\partial x_{i}}{\partial \theta_{7}} & \frac{\partial x_{i}}{\partial \theta_{8}} \\ \frac{\partial y_{i}}{\partial x} & \frac{\partial y_{i}}{\partial y} & \frac{\partial y_{i}}{\partial \theta} & \frac{\partial y_{i}}{\partial \theta_{1}} & \frac{\partial y_{i}}{\partial \theta_{2}} & \frac{\partial y_{i}}{\partial \theta_{3}} & \frac{\partial y_{i}}{\partial \theta_{4}} & \frac{\partial y_{i}}{\partial \theta_{5}} & \frac{\partial y_{i}}{\partial \theta_{6}} & \frac{\partial y_{i}}{\partial \theta_{7}} & \frac{\partial y_{i}}{\partial \theta_{8}}\end{array}\right]$

Namlunun gövdeye etkisini bulmak için kullanılması gereken jakobiyen matrisi de Ek-3'de verilmiştir. Bu çalışmada ele alınan 4 bacaklı robot ve üzerine yerleştirilen silah kulesine ait parametreler Tablo 2'de verilmiştir.

Tablo 2. Robotun Fiziksel Parametrelerinin Sayısal Değerleri

\begin{tabular}{cc}
\hline Parametre & Değer \\
\hline$M$ & $12 \mathrm{~kg}$ \\
$l_{g}$ & $0.8 \mathrm{~m}$ \\
$l_{1}, l_{2}, l_{5}, l_{6}$ & $0.24 \mathrm{~m}$ \\
$l_{3}, l_{4}, l_{7}, l_{8}$ & $0.2 \mathrm{~m}$ \\
$m_{1}, m_{2}, m_{5}, m_{6}$ & $1.5 \mathrm{~kg}$ \\
$m_{3}, m_{4}, m_{7}, m_{8}$ & $0.5 \mathrm{~kg}$ \\
$\mathrm{I}$ & $0.64 \mathrm{kgm}^{2}$ \\
$\mathrm{a}$ & $0.1 \mathrm{~m}$ \\
$\mathrm{~b}$ & $0.3 \mathrm{~m}$ \\
$m_{n}$ & $1 \mathrm{~kg}$ \\
\hline
\end{tabular}

\section{Robotun Kontrolü ve Yürüyüş Simülasyonları}

Klasik PID kontrol yöntemi kapalı çevrim kontrol yapısına sahiptir. Kapalı çevrim kontrol yapısına geri beslemeli kontrol yapısı adı da verilmektedir. PID kontrol yöntemine göre kontrol işaret sinyali (24)'te görüldügü gibi hesaplanmaktadır.

$u(t)=K_{p} e(t)+K_{d} \frac{d e(t)}{d_{t}}+K_{i} \int_{0}^{t} e(t) d_{t}$

Burada $K_{p}$; oransal kazanc1, $K_{d}$; türevsel kazanc1, $K_{i}$; integral kazanc1, e(t) ise hata değerini ifade etmektedir. Oransal kazanç; kontrol hatasının anlık değerlerine bağlı bir katkı sağlamaktadır. Türevsel kazanç; kontrol hatasının değişme oranına etki etmekte ve sonuç olarak hatayı sıfıra götüren hızlı bir mod biçimidir. İntegral kazanç; birikmiş hatayla orantılı olan ve yavaş bir kontrol modu anlamına gelen bir kontrolör çıkışı vermekte, sistemin kalıcı durum hatasını sıfıra zorlamaktadır [12]. Bu çalışmada tasarlanan kontrolörde oransal, türevsel ve integral katsayıları deneme yanılma tekniği ile $K_{p}=500$, $K_{d}=50, K_{i}=0.1$ olarak belirlenmiştir.

Robota ait her bir bacak ekleminin izlemesi gereken açısal referans konumları, yörünge planlaması ile belirlenmiştir. Daha sonra eklemler PID kontrol ile bu referans konumlara götürülmek üzere kontrol edilmiştir. Bacak hareket mekanizması çok karmaşık bir problemdir. Her bir bacak ileriye ve geriye doğru hareket etmektedir. Böceklerin hareketinden türetilen adım hareketi, bacak havada(flight) ve bacak yerde(stance) olmak üzere 2 aşamaya sahiptir [13]. Dört Bacaklı(Quadruped) robotlar, 4 tip yürüyüşe sahiptir. Bu yürüyüşlere literatürde walk, trot, pace ve gallop isimleri verilmiştir [14]. Bu çalışmada simülasyonlar için Şekil 2'de gösterilen trot yürüyüş dizisi kullanılmıştır [15]. Bu yürüyüş dizisinin ilk yarı periyodunda robotun sol ön bacağı ile sağ arka bacağı yer ile temas halindedir. Yürüyüş dizisinin son yarı periyodunda ise robotun sağ ön bacağı ile sol arka bacağı yer ile temas 
halindedir. Bu yürüyüş dizisinin robot gövdesinde yol açtığı hareketlilik namlu stabilizasyonuna bozucu etki olarak yansımaktadır.

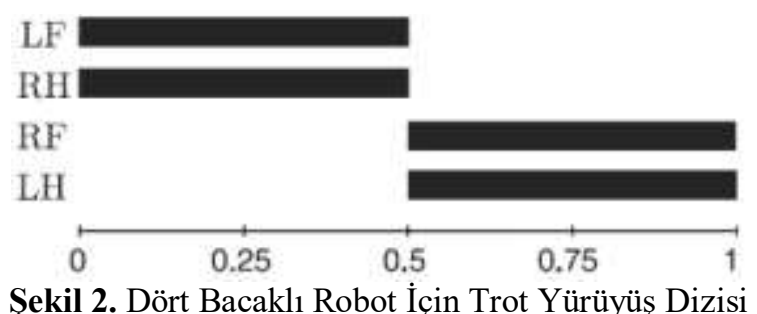

Trot yürüyüşü esnasında bir bacak adım atarken ayak ucu Şekil 3’te x-y düzleminde gösterilen eğri yolunu takip etmektedir. Her bir adım havada ve yerde olan aşamalardan oluşmaktadır. Bacağın yer ile temasının kesildiği anda havadaki aşama başlamaktadır. Bacaklar hareketini havadayken sinüzoidal olarak gerçekleştirmektedir. Bacağın yere temas etmeye başladığı anda havadaki aşama bitmekte ve yerdeki aşama başlamaktadır. Yerdeki aşamada bacak yere sürekli temas etmektedir ve ayak ucu zemine paralel olarak düz doğrusal hareket ettirilmektedir. Bu sayede yerdeki aşamada robot gövdesi bacak tarafindan yere paralel biçimde itilmektedir. Dört bacaklı robot trot yürüyüş periyodu boyunca daima 2 bacak üzerinde taşınmaktadır.

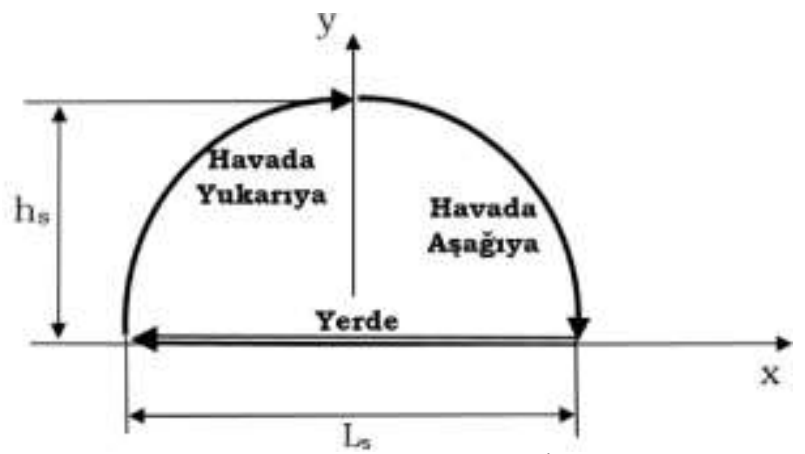

Şekil 3. Robot Bacağının Bir Adımında İzleyeceği Yörünge

Bacağın havadaki aşamasında izlemesi gereken yörüngeyi matematiksel olarak ifade edebilmek için (25)'te verilen sinüzoidal fonksiyon kullanılmıştır. Burada $h_{\text {step }}$ bacağın yerden kalkma yüksekliğidir. Fonksiyona ait $\omega$ parametresi, adım hareketinin frekansını ifade etmektedir.

$h_{s}=h_{\text {step }} \operatorname{Sin}(2 \pi \omega t)$

Yer (stance) aşamasında $L_{s}$ olarak belirtilen kenar uzunluğu, bacağın bir adımında x-ekseni doğrultusundaki ilerleme miktarını göstermektedir. Yer aşamasında geçen süre, bir periyotluk yürüyüş için geçen toplam sürenin $\frac{1}{2}$ 'sine eşittir. Yürüyüşün bir periyotluk toplam süresi $T_{G}$ saniye olarak gösterilirse robotun ilerleme hızı $V_{r}$ (26) ifadesinde görüldügü gibi $L_{S}$ adım uzunluğuna bağlı olarak hesap edilebilmektedir. Adım uzunluğu, adım yüksekliği ve yürüyüş periyodu gibi parametrelerin değiştirilmesi ile adım karakteristikleri düzenlenebilmektedir.

$V_{r}=\frac{L_{S}}{T_{G}}$

Dinamik modeli elde edilmiş dört bacaklı robotun trot yürüyüşü Matlab'da simüle edilerek gerçekleştirilmiştir. Yürüyüş simülasyonunun farklı aşamalarından elde edilmiş görüntü Şekil 4'te verilmiştir. 


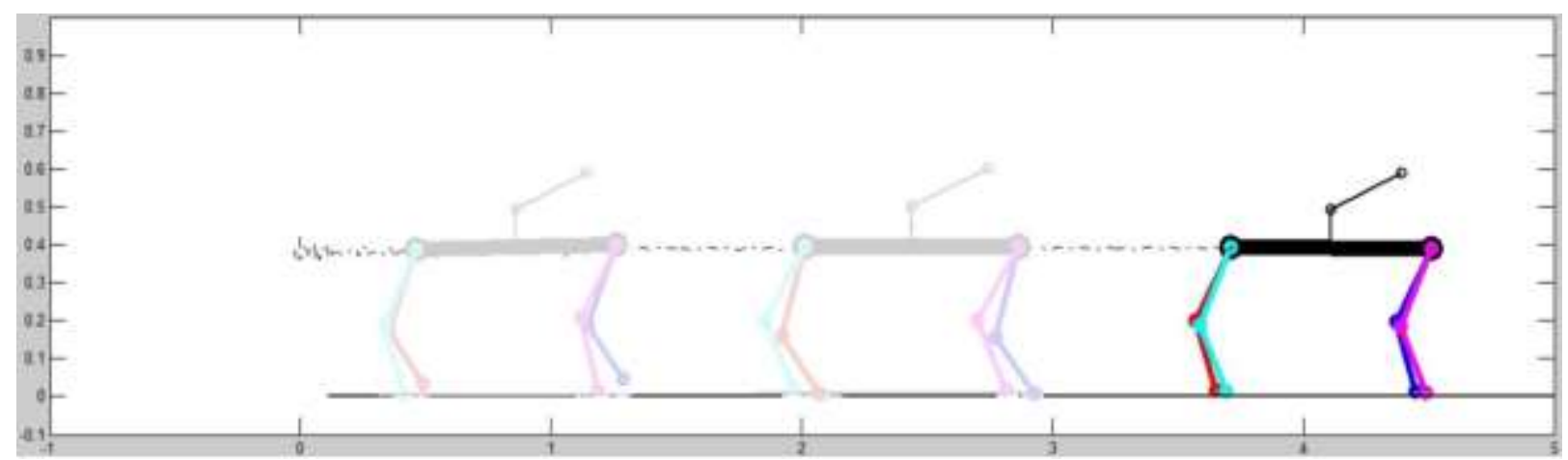

Şekil 4. Dört Bacaklı Robotun Trot Yürüyüş Simülasyonu
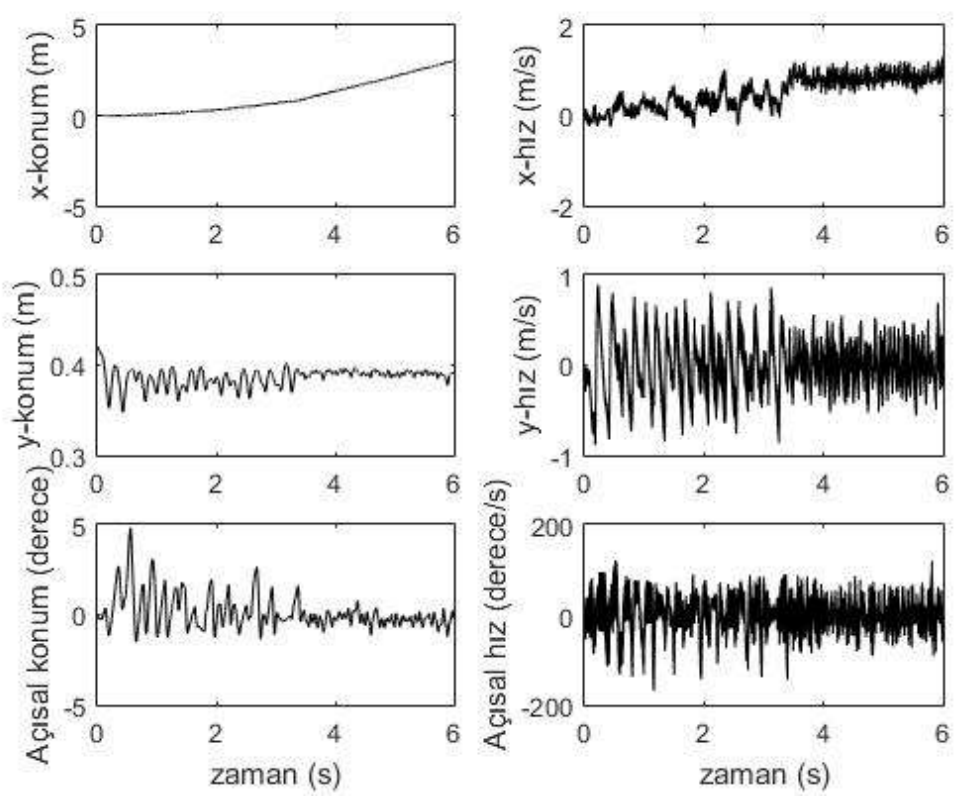

Şekil 5. Robotun Trot Yürüyüş Simülasyonundan Elde Edilen Konum ve Hız Grafikleri

Robot durağan halden harekete başlamış ve 6 saniyelik simülasyon sonunda yaklaşık 4 metre kadar yol almıştır. Simülasyon süresince robot gövde merkezinin yatay $\mathrm{x}$ - ekseninde ve düşey $\mathrm{y}$ eksenindeki zamana göre yer değişimleri, düzleme dik eksen etrafındaki açısal yer değişimi Şekil 5.'te gösterilmiştir. Bu grafiklerle birlikte ayrıca robot gövde merkezinin doğrusal ve açısal hız değişimlerini gösteren grafikler de sunulmuştur. Yürüyüşün ilk anlarında robot gövde merkezinin düşeydeki iniş ve kalkış biçimindeki salınımının genliği 3-4 cm dolaylarında olduğu görülmektedir. Aynı biçimde robot gövde merkezinin yalpalama genliği ise 2-3 derece dolaylarında olduğu görülmüştür. Simülasyonun 3. saniyesinden itibaren bu salınım ve yalpalama genliklerinde büyük oranda azalmalar oluşmuştur. Bununla birlikte robot, simülasyonun 4. saniyesinden itibaren $1 \mathrm{~m} / \mathrm{s}$ büyüklügünde doğrusal hiza sahip olarak yatayda ilerlemeye devam etmektedir.

Şekil 5'teki grafiklere bakıldığında, robotun kendi gövdesini düşey eksende yaklaşık $0.4 \mathrm{~m}$ yükseklikte tutmaya çalıştığı görülmektedir. Dolayısıyla, düşey eksendeki hızı yaklaşık $0 \mathrm{~m} / \mathrm{s}$ dir. Robotun yatay eksendeki hareketine bakıldığında ise simülasyon değerlerinde verildiği gibi 4 m'lik yolu $1 \mathrm{~m} / \mathrm{s}$ hız ile aldığg görülmektedir.

\section{Kayan Kipli Kontrol Yöntemi ile Silah Kulesinin Stabilizasyonu}

Silah kulesi kontrol sistemi, atış kontrol sistemlerinin taktiksel ve teknik performansının zorunlu bir parçasıdır. Kayan kipli kontrol, istenen hedef açısına göre hedefin etkin bir biçimde izlenmesinde yaygın olarak kullanılmaktadır [16]. Kayan kipli kontrol yöntemi yüksek hızlı, doğrusal olmayan bir geri besleme ile önceden belirlenen bir kayma yüzeyi üzerinde zamanda süreksiz bir şekilde anahtarlama 
yapılarak elde edilen, belirgin, doğrusal olmayan, dayanıklı bir kontrol yöntemidir [17]. Kayan kipli kontrol tasarımı iki aşamalı bir sürece sahiptir. Bu aşamalar sırasıyla; kayma yüzeyinin belirlenmesi ve belirlenen kayma yüzeyine ulaşmayı sağlayan bir kontrol kuralının elde edilmesidir. Kontrol kuralı sistemin dinamiklerini kayma yüzeyine ulaşmaya zorlamaktadır. Sistem dinamikleri kayma yüzeyine ulaştı̆̆ında kayma yüzeyinde tutulmaya zorlamaktadır. Bu esnada kayma kipi oluşmaktadır. Kayma kontrolde sistem, parametre belirsizlikleri ve dış bozuculara karşı duyarsız davranmaktadır [18]. Kayan kipli kontrol uygulamalarından meydana gelen çatırtı problemi, sistemin ulaşmak istediği denge noktası etrafinda meydana gelen osilasyonlardan kaynaklanır ve sistemin modellenmemiş yüksek frekanslı dinamiklerini ortaya çıkarmaktadır.

Bir işaret fonksiyonuna sahip kayan kipli kontrol ifadesi (27)'deki gibi yazılabilir.

$U=-K_{s m c} \operatorname{Sign}(S)$

Burada S ifadesi kayma yüzeyi fonksiyonudur ve sistem cevabından alınan hata (e) ve hatanın zamana göre değişimine (de) bağlı olarak aşağıdaki gibi yazılabilir.

$S=C_{s m c} e+d e$

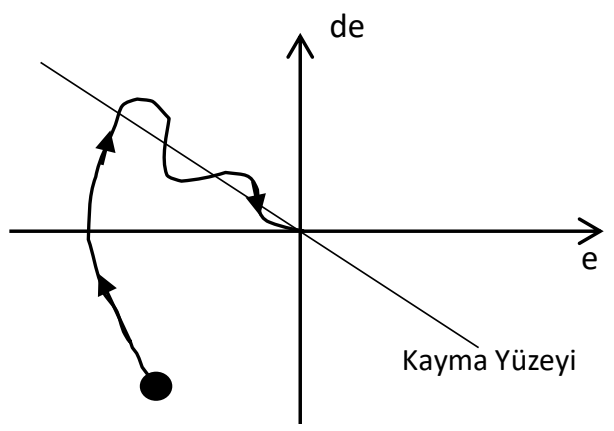

Şekil 6. Kayma Yüzeyi

Kayan kipli kontrolün (27) ifadesinde yer alan sign fonksiyonu sinyalin çatırdamasına yol açmaktadır. Bu çatırdamalar sistem üzerinde olumsuz etkilere yol açmaktadır. Çatırdama problemini azaltmaya yönelik bir saturasyon fonksiyonu kullanılarak kayan kipli kontrol ifadesi;

$U=-K_{s m c} \operatorname{Sat}\left(\frac{S}{\varnothing}\right)$

olarak yazılabilir. Burada $\phi$ ifadesi sınır tabaka kalınlığıdır. Buna göre saturasyon fonksiyonu (29) ifadesindeki gibi hesap edilebilmektedir.

$\operatorname{Sat}\left(\frac{S}{\varnothing}\right)=\left\{\begin{array}{cc}1 & \left(\frac{S}{\varnothing}\right)>1 \\ -1 & \left(\frac{S}{\varnothing}\right)<-1 \\ \left(\frac{S}{\varnothing}\right) & -1<\left(\frac{S}{\varnothing}\right)<1\end{array}\right\}$

Bu çalışmada robot üzerine yerleştirilmiş silah kulesinin namlu açısı, hedeflenen konumda tutulmak istenmiştir. Robotun yürümesi sonucu gövdesi üzerinde oluşan salınım ve yalpalama hareketleri silah kulesine bozucu bir etki olarak tesir etmektedir. Namluyu hedeflenen açısal konumda tutabilmek için kayan kipli kontrol yöntemi uygulanmıştır. Namlu için belirlenen kontrol kazanç katsayıları $K_{s m c}=50, C_{s m c}=65, \phi=0.8$ olarak belirlenmiştir. Bu katsayılar deneme yanımla tekniği ile elde edilmiştir. Bu çalışmada namlunun 3 farklı hedef açısı için robot yürüyüşü esnasında kontroller gerçekleştirilmiş̧ir. Simülasyonlarda namlu başlangıç açısı sıfır olarak kabul edilmiştir. 
Referans Açı-1;

Namlu açısının yere göre $15^{\circ}$ de tutulmak istenmesi ile uygulanan kontrol ve gerçekleştirilen simülasyonun sistem cevapları Şekil 7.'de grafiksel olarak sunulmuştur. Namlunun gövdeye göre bağıl açısal konumu $\alpha$, gövde hareketleri ile ilişkili olarak salınımlı olduğu görülmüştür. Kontrolör, namlunun yatay x-eksenine göre mutlak açısal konumunu $(\alpha+\theta)$, oldukça küçük hata ve salınımlarla hedef açısında tutulabilmiştir. Referans konum etrafında namlunun açısal hata büyüklügü en fazla $0.12^{\circ}$ olarak elde edilmiştir.
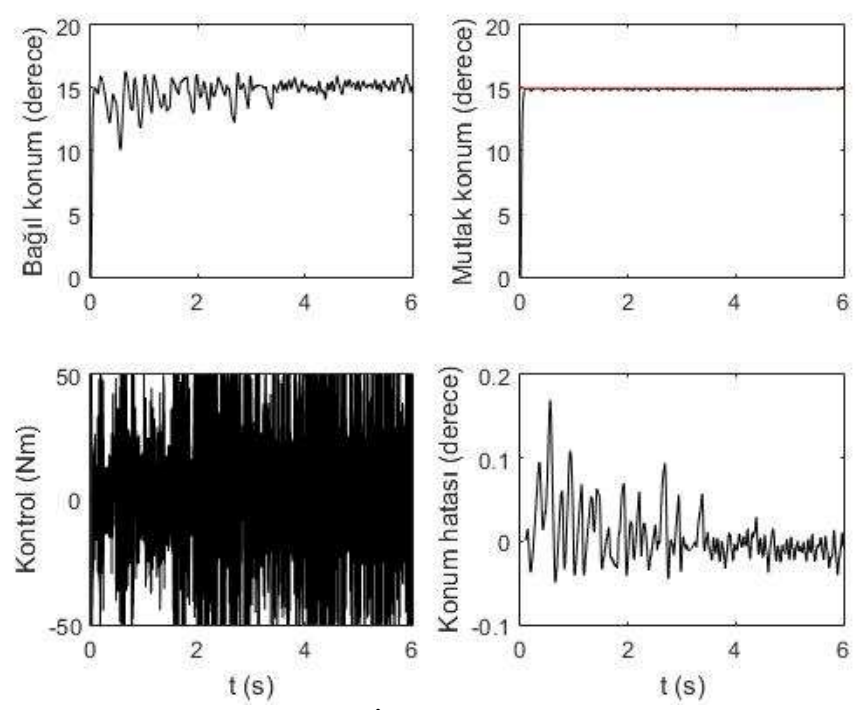

Şekil 7. Simülasyonda $15^{\circ}$ İçin Namluya Ait Sistem Cevapları

Referans Aç1-2;

Namlu açısının yere göre $\underline{35^{\circ}}$ de tutulmak istenmesi ile uygulanan kontrol ve gerçekleştirilen simülasyonun sistem cevapları Şekil 8.'de grafiksel olarak sunulmuştur. Namlu açısal konumunun, referans açısal konumu küçük bir miktar hata payı ile izleyebildiği görülmüştür. Referans konum etrafinda namlunun açısal hata büyüklügü en fazla $0.15^{\circ}$ olarak elde edilmiştir.
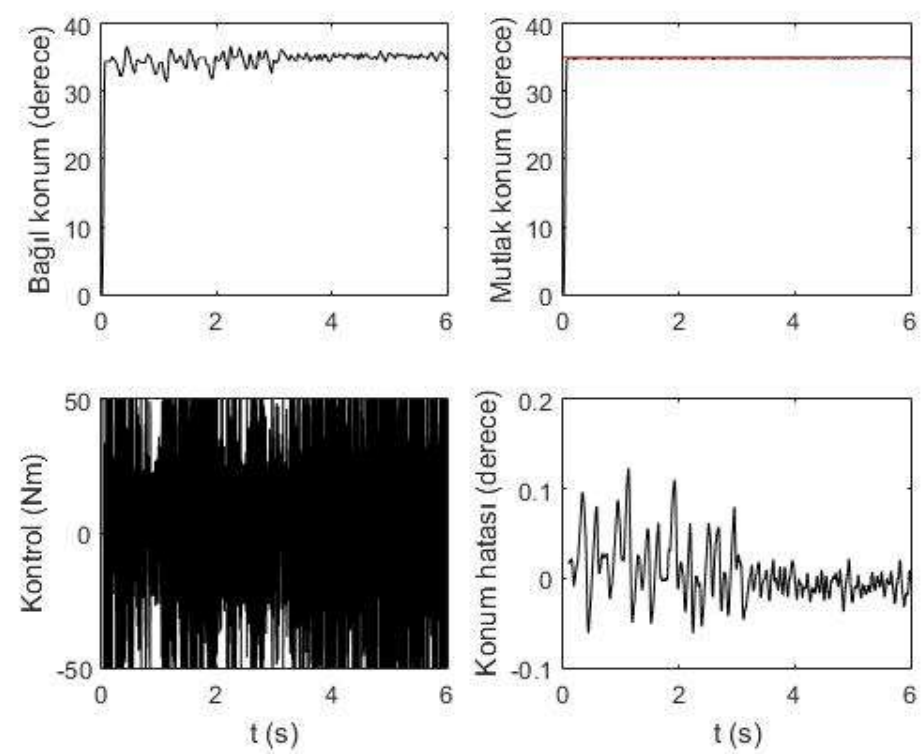

Şekil 8. Simülasyonda $35^{\circ}$ İçin Namluya Ait Sistem Cevapları

Referans Aç1-3;

Namlu açısının yere göre $50^{\circ}$ de tutulmak istenmesi ile uygulanan kontrol ve gerçekleştirilen simülasyonun sistem cevapları Şekil 9.'da grafiksel olarak sunulmuştur. Namlu açısal konumunun, 
referans açısal konumu izlerken referans konum etrafindaki açısal hata büyüklügünün en fazla $0.15^{\circ}$ olduğu görülmüştür.
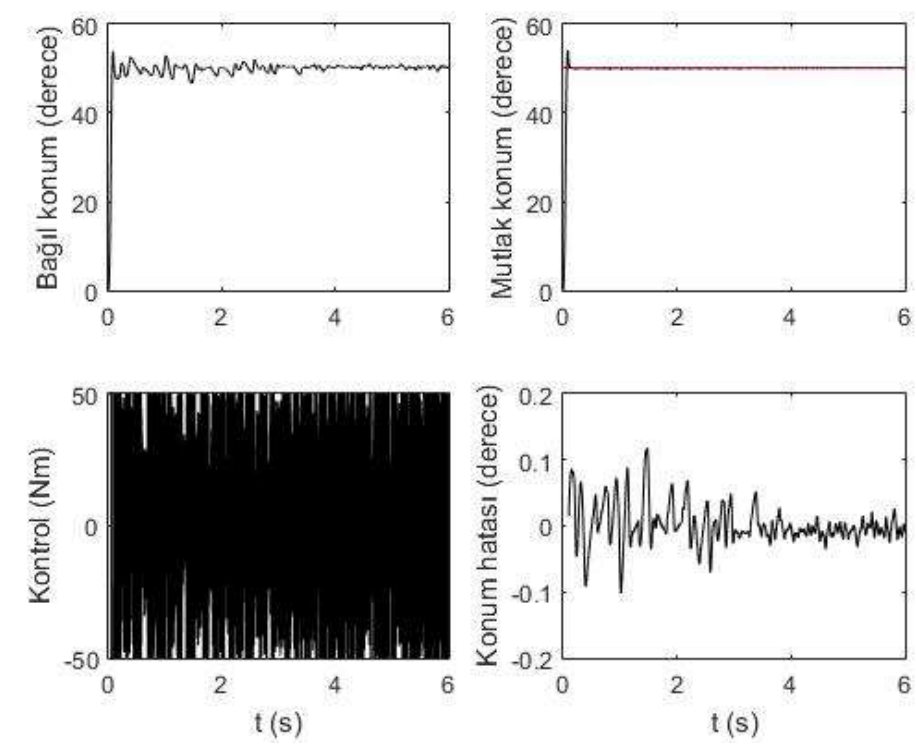

Şekil 9. Simülasyonda $50^{\circ}$ İçin Namluya Ait Sistem Cevapları

Her üç referans konum açısı için elde edilen namlu açısal hatası, büyüklük olarak birbirine oldukça yakın çıkmıştır. Bu hata miktarı robotun yürüyüşü esnasında gövdesinde oluşan salınımlı hareketlerin miktarına bağlı olarak değişebilmektedir. Simülasyonlarda kullanılan kayan kipli kontrolörün, namluyu referans konum etrafında, oldukça başarılı bir şekilde tutabildiği, sunulan grafiklerden görülmektedir.

\section{Sonuçlar}

$\mathrm{Bu}$ çalışmada namlu stabilizasyonu için dört ayaklı bir robot üzerine bir silah kulesi yerleştirilmiştir. Düzlemsel olarak ele alınan sistemin dinamik hareket denklemleri matematiksel olarak elde edilmiştir. Robotun trot yürüyüşü sağlanarak simülasyonlar gerçekleştirilmiştir. $\mathrm{Bu}$ yürüyüş esnasında robot gövdesinde meydana gelen yalpa ve salınım hareketleri silah kulesine bozucu etki olarak tesir ettirilmiştir. Namlunun stabilizasyonu için kayan kipli kontrol yöntemi uygulanmıştır. Kontrol kazanç katsayıları deneme yanılma tekniği ile elde edilmiştir. Simülasyonlar neticesinde elde edilen sistem cevaplarında namlunun referans konumlarını belirli bir hata ile oldukça başarılı bir biçimde kontrol edebildiği görülmüsşür. Ancak kontrol kazanç katsayılarının optimum değerlerinin kullanımı ile bu başarının artacağı beklenmektedir. Bununla birlikte farklı kontrol yöntemlerinin uygulanması ve bu kontrol yöntemlerinin karşılaştırılması ile bu başarının sonuçları ortaya konulabilecektir. Bu çalışmada üzerine silah kulesi yerleştirilmiş dört ayaklı bir robotun savunma sanayisinde kullanılabileceği ve namlu stabilizasyonunun kayan kipli kontrol ile gerçekleştirilebileceği ortaya konulmuştur.

\section{Kaynaklar}

[1] Işsk H. 2016. Namlu İçerisindeki Balistik Parametrelerin Modellenmesi. The Journal of Defense Sciences, 15: 159 .

[2] Afacan K. 2004. Bir Stabilizasyon Sisteminin Modellenmesi ve Kontrolü. Ortadoğu Teknik Üniversitesi, Fen Bilimleri Enstitüsü, Yüksek Lisans Tezi, 89s, Ankara.

[3] Songül S. 2014. Tank Namlusu Stabilizasyon Sisteminin Arduino ile Uygulanması ve Deneysel Düzeneğinin Hazırlanması. Trakya Üniversitesi, Fen Bilimleri Enstitüsü, Yüksek Lisans Tezi, 66s, Edirne.

[4] Gümüşay Ö. 2006. Herhangi Bir Araziden Gelen Bozucu Etkiler Altındaki Taret Alt Sistemlerinin Akıllı Stabilizasyon Denetimi. Ortaduğu Teknik Üniversitesi, Fen Bilimleri Enstitüsü, Yüksek Lisans Tezi, 167s, Ankara. 
[5] Korobiichuk I. 2016. Mathematical Model of Presicion Sensor for an Automatic Weapons Stabilizer System. Measurement, 89: 151-158.

[6] Kline A., Ahner D., Hill R. 2019. The Weapon-Target Assignement Problem. Computer and Operations Research, 105: 226-236.

[7] Gor M.M., Pathak P.M., Samantaray A.K., Yang J.M., Kwak S.W. 2015. Control Oriented Model-Based Simulation and Experimental Studies on a Compliant Legged Quadruped Robot. Robotics and Autonomous Systems, 72: 217-234.

[8] Ganesh K.K., Pathak P.M. 2013. Dynamic Modelling \& Simulation of a Four Legged Jumping Robot with Compliant Legs. Robotics and Autonomous Systems, 61: 221-228.

[9] Gao, Z., Shi, Q., Fukuda, T., Li, C., Huang, Q. 2019. An overview of biomimetic robots with animal behaviours. Neurocomputing, 332: 339-350.

[10] Dhaoudai R., Hatab A.A. 2013. Dynamic Modelling of Differential-Drive Mobile Robots Using Lagrange and Newton-Euler Methodologies: A Unified Framework. Advanced in Robotics and Automation, 2: 3.

[11] Beckers T., Kulić D., Hirche S. 2019. Stable Gaussian process based tracking control of EulerLagrange systems. Automatica, 103: 390-397.

[12] Goodwin C.G., Graebe S.F., Salgado M.E. 2000. Control System Design. Prentice Hall, pp: 158159.

[13] Jakimovski B. 2011. Biologically Inspired Approaches for Locomotion, Anomaly Detection and Reconfiguration for Walking Robots. Springer-Verlag Berlin Heidelberg.

[14] Pei Z., Song L., Chen B., Guo X. 2012. Adaptive Control of a Quadruped Robot Based on Central Pattern Generators, IEEE.

[15] Santos C.P., Matos V. 2011. Gait Transition and Modulation in a Quadruped Robot: A BrainstemLike Modulation Approach. Robotics and Autonomous Systems, 59: 620-634.

[16] Rahmat M.S., Hudha K., Idris A.M., Amer N.H. 2016. Sliding Mode Control of Target Tracking System for Two Degrees of Freedom Gun Turret Model. Advanced in Military Technology, 11 (1): $13-28$.

[17] Young K.D., Utkin V.I., Ozguner U. 1999. A Control Engineer's Guide to Sliding Mode Control. IEEE Transactions on Control Systems Technology, 7 (3): 328-342.

[18] Edwards C., Spurgeon S.K. 1998. Sliding Mode Control. Theory and Applications, London, Taylor and Francis. 


\section{Ekler}

1.

$L=\frac{1}{2} M \dot{x}^{2}+\frac{1}{2} M \dot{y}^{2}+\frac{1}{2} I \dot{\theta}^{2}+\frac{m_{1} \dot{x}^{2}}{2}+\frac{m_{1} \dot{y}^{2}}{2}+\frac{m_{1} L^{2} \dot{\theta}^{2}}{8}+\frac{m_{1} L_{1}^{2} \dot{\theta}_{1}^{2}}{2}+\frac{m_{1} L \dot{x} \dot{\theta} \sin \theta}{2}-m_{1} \dot{x}_{1} \dot{\theta}_{1} \sin \theta_{1}$ $-\frac{m_{1} L \dot{y} \dot{\theta} \cos \theta}{2}+m_{1} \dot{y} L_{1} \dot{\theta}_{1} \cos \theta_{1}-\frac{m_{1} L L_{1} \dot{\theta} \dot{\theta}_{1} \cos \left(\theta-\theta_{1}\right)}{2}+\frac{m_{2} \dot{x}^{2}}{2}+\frac{m_{2} \dot{y}^{2}}{2}+\frac{m_{2} L^{2} \dot{\theta}^{2}}{8}+\frac{m_{2} L_{2}^{2} \dot{\theta}_{2}^{2}}{2}$ $-\frac{m_{2} L \dot{x} \dot{\theta} \sin \theta}{2}-m_{2} \dot{x} L_{2} \dot{\theta}_{2} \sin \theta_{2}+m_{2} \dot{y} L_{2} \dot{\theta}_{2} \cos \theta_{2}+\frac{m_{2} L \dot{y} \dot{\theta} \cos \theta}{2}+\frac{m_{2} L L_{2} \dot{\theta} \dot{\theta}_{2} \cos \left(\theta-\theta_{2}\right)}{2}+\frac{m_{3} \dot{x}^{2}}{2}$ $+\frac{m_{3} \dot{y}^{2}}{2}+\frac{m_{3} L^{2} \dot{\theta}^{2}}{8}+\frac{m_{3} L_{1}^{2} \dot{\theta}_{1}^{2}}{2}+\frac{m_{3} L_{3}^{2}\left(\dot{\theta}_{1}+\dot{\theta}_{3}\right)^{2}}{2}+\frac{m_{3} L \dot{x} \dot{\theta} \sin \theta}{2}-m_{3} \dot{x}_{1} \dot{\theta}_{1} \sin \theta_{1}$

$-m_{3} \dot{x} L_{3}\left(\dot{\theta}_{1}+\dot{\theta}_{3}\right) \sin \left(\theta_{1}+\theta_{3}\right)+m_{3} \dot{y} L_{1} \dot{\theta}_{1} \cos \theta_{1}-\frac{m_{3} L \dot{y} \dot{\theta} \cos \theta}{2}+m_{3} \dot{y} L_{3}\left(\dot{\theta}_{1}+\dot{\theta}_{3}\right) \cos \left(\theta_{1}+\theta_{3}\right)$ $-\frac{m_{3} L L_{1} \dot{\theta} \dot{\theta}_{1} \cos \left(\theta-\theta_{1}\right)}{2}-\frac{m_{3} L L_{3} \dot{\theta}\left(\dot{\theta}_{1}+\dot{\theta}_{3}\right) \cos \left(\theta-\theta_{1}-\theta_{3}\right)}{2}+m_{3} L_{1} L_{3} \dot{\theta}_{1}\left(\dot{\theta}_{1}+\dot{\theta}_{3}\right) \cos \theta_{3}+\frac{m_{4} \dot{x}^{2}}{2}$ $+\frac{m_{4} \dot{y}^{2}}{2}+\frac{m_{4} L^{2} \dot{\theta}^{2}}{8}+\frac{m_{4} L_{2}^{2} \dot{\theta}_{2}^{2}}{2}+\frac{m_{4} L_{4}^{2}\left(\dot{\theta}_{2}+\dot{\theta}_{4}\right)^{2}}{2}-\frac{m_{4} L \dot{x} \dot{\theta} \sin \theta}{2}-m_{4} \dot{x} L_{4}\left(\dot{\theta}_{2}+\dot{\theta}_{4}\right) \sin \left(\theta_{2}+\theta_{4}\right)$ $-m_{4} \dot{x} L_{2} \dot{\theta}_{2} \sin \theta_{2}+\frac{m_{4} L \dot{y} \dot{\theta} \cos \theta}{2}+\frac{m_{4} L L_{2} \dot{\theta} \dot{\theta}_{2} \cos \left(\theta-\theta_{2}\right)}{2}+\frac{m_{4} L L_{4} \dot{\theta}\left(\dot{\theta}_{2}+\dot{\theta}_{4}\right) \cos \left(\theta-\theta_{2}-\theta_{4}\right)}{2}$ $+m_{4} L_{2} L_{4} \dot{\theta}_{2}\left(\dot{\theta}_{2}+\dot{\theta}_{4}\right) \cos \theta_{4}+\frac{m_{5} \dot{x}^{2}}{2}+\frac{m_{5} \dot{y}^{2}}{2}+\frac{m_{5} L^{2} \dot{\theta}^{2}}{8}+\frac{m_{5} L_{5}^{2} \dot{\theta}_{5}^{2}}{2}+\frac{m_{5} L \dot{x} \dot{\theta} \sin \theta}{2}-\frac{m_{5} L \dot{y} \dot{\theta} \cos \theta}{2}$ $-m_{5} \dot{x} L_{5} \dot{\theta}_{5} \sin \theta_{5}+m_{5} \dot{y} L_{5} \dot{\theta}_{5} \cos \theta_{5}-\frac{m_{5} L L_{5} \dot{\theta} \dot{\theta}_{5} \cos \left(\theta-\theta_{5}\right)}{2}+\frac{m_{6} \dot{x}^{2}}{2}+\frac{m_{6} \dot{y}^{2}}{2}+\frac{m_{6} L^{2} \dot{\theta}^{2}}{8}+\frac{m_{6} L_{6}^{2} \dot{\theta}_{6}^{2}}{2}$ $-\frac{m_{6} L \dot{x} \dot{\theta} \sin \theta}{2}-m_{6} \dot{x} L_{6} \dot{\theta}_{6} \sin \theta_{6}+\frac{m_{6} L \dot{y} \dot{\theta} \cos \theta}{2}+m_{6} \dot{y} L_{6} \dot{\theta}_{6} \cos \theta_{6}+\frac{m_{7} \dot{x}^{2}}{2}+\frac{m_{7} \dot{y}^{2}}{2}+\frac{m_{7} L^{2} \dot{\theta}^{2}}{8}$ $+\frac{m_{7} L_{5}^{2} \dot{\theta}_{5}^{2}}{2}+\frac{m_{7} L_{7}^{2}\left(\dot{\theta}_{5}+\dot{\theta}_{7}\right)^{2}}{2}+\frac{m_{7} L \dot{x} \dot{\theta} \sin \theta}{2}-m_{7} \dot{x}_{5} \dot{\theta}_{5} \sin \theta_{5}-m_{7} \dot{x}_{7}\left(\dot{\theta}_{5}+\dot{\theta}_{7}\right) \sin \left(\theta_{5}+\theta_{7}\right)$ $-\frac{m_{7} L \dot{y} \dot{\theta} \cos \theta}{2}+m_{7} \dot{y} L_{5} \dot{\theta}_{5} \cos \theta_{5}+m_{7} \dot{y} L_{7}\left(\dot{\theta}_{5}+\dot{\theta}_{7}\right) \cos \left(\theta_{5}+\theta_{7}\right)-\frac{m_{7} L L_{5} \dot{\theta} \dot{\theta}_{5} \cos \left(\theta-\theta_{5}\right)}{2}$ $-\frac{m_{7} L L_{7} \dot{\theta}\left(\dot{\theta}_{5}+\dot{\theta}_{7}\right) \cos \left(\theta-\theta_{5}-\theta_{7}\right)}{2}+m_{7} L_{5} L_{7} \dot{\theta}_{5}\left(\dot{\theta}_{5}+\dot{\theta}_{7}\right) \cos \theta_{7}+\frac{m_{8} \dot{x}^{2}}{2}+\frac{m_{8} \dot{y}^{2}}{2}+\frac{m_{8} L^{2} \dot{\theta}^{2}}{8}$ $+\frac{m_{8} L_{6}^{2} \dot{\theta}_{6}^{2}}{2}+\frac{m_{8} L_{8}^{2}\left(\dot{\theta}_{6}+\dot{\theta}_{8}\right)^{2}}{2}-\frac{m_{8} L \dot{x} \dot{\theta} \sin \theta}{2}-m_{8} \dot{x} L_{8}\left(\dot{\theta}_{6}+\dot{\theta}_{8}\right) \sin \left(\theta_{6}+\theta_{8}\right)+m_{8} \dot{y} L_{6} \dot{\theta}_{6} \cos \theta_{6}$ $-m_{8} \dot{x} L_{6} \dot{\theta}_{6} \sin \theta_{6}+\frac{m_{8} L \dot{y} \dot{\theta} \cos \theta}{2}+m_{8} \dot{y} L_{8}\left(\dot{\theta}_{6}+\dot{\theta}_{8}\right) \cos \left(\theta_{6}+\theta_{8}\right)+\frac{m_{8} L L_{6} \dot{\theta} \dot{\theta}_{6} \cos \left(\theta-\theta_{6}\right)}{2}$ $+m_{8} L_{6} L_{8} \dot{\theta}_{6}\left(\dot{\theta}_{6}+\dot{\theta}_{8}\right) \cos \theta_{8}+\frac{m_{8} L L_{8} \dot{\theta}\left(\dot{\theta}_{6}+\dot{\theta}_{8}\right) \cos \left(\theta-\theta_{6}-\theta_{8}\right)}{2}+\frac{m_{n} \dot{x}^{2}}{2}+\frac{m_{n} \dot{y}^{2}}{2}+\frac{m_{n} \mathrm{a}^{2} \dot{\theta}^{2}}{2}$ $+\frac{m_{n} \mathrm{~b}^{2}(\dot{\alpha}+\dot{\theta})^{2}}{2}-m_{n} \dot{x} \mathrm{a} \dot{\theta} \cos \theta-m_{n} \dot{x} \mathrm{~b}(\dot{\alpha}+\dot{\theta}) \sin (\alpha+\theta)-m_{n} \dot{y} \mathrm{a} \dot{\theta} \sin \theta+m_{1} g \frac{L}{2} \sin \theta$ )$+m_{n} \mathrm{ab} \dot{\theta}(\dot{\alpha}+\dot{\theta}) \sin \alpha-M g y-m_{1} g y-m_{1} g L_{1} \sin \theta_{1}-m_{2} g y-m_{2} g \frac{L}{2} \sin \theta-m_{2} g L_{2} \sin \theta_{2}$ $-m_{3} g y+m_{3} g \frac{L}{2} \sin \theta-m_{3} g L_{1} \sin \theta_{1}-m_{3} g L_{3} \sin \left(\theta_{1}+\theta_{3}\right)-m_{4} g y+m_{4} g \frac{L}{2} \sin \theta$ $-m_{4} g L_{2} \sin \theta_{2}-m_{4} g L_{2} \sin \left(\theta_{2}+\theta_{4}\right)-m_{5} g y+m_{5} g \frac{L}{2} \sin \theta-m_{5} g L_{5} \sin \theta_{5}-m_{6} g y$ $-m_{6} g \frac{L}{2} \sin \theta-m_{6} g L_{6} \sin \theta_{6}-m_{7} g y+m_{7} g \frac{L}{2} \sin \theta-m_{7} g L_{5} \sin \theta_{5}-m_{8} g y+m_{8} g \frac{L}{2} \sin \theta$ $-m_{7} g L_{7} \sin \left(\theta_{5}+\theta_{7}\right)-m_{8} g L_{6} \sin \theta_{6}-m_{n} g y-m_{n} g a \sin (\alpha+\theta)-m_{n} g a \cos \theta$

2. $\sum \boldsymbol{F}_{\boldsymbol{x}}=M \ddot{x}+m_{1} \ddot{x}+\frac{m_{1} L \ddot{\theta} \sin \theta}{2}+\frac{m_{1} L \dot{\theta}^{2} \cos \theta}{2}-m_{1} L_{1} \ddot{\theta}_{1} \sin \theta_{1}-m_{1} L_{1} \dot{\theta}_{1}^{2} \cos \theta_{1}+m_{2} \ddot{x}-\frac{m_{2} L \ddot{\theta} \sin \theta}{2}$ $-\frac{m_{2} L \dot{\theta}^{2} \cos \theta}{2}-m_{2} L_{2} \ddot{\theta}_{2} \sin \theta_{2}-m_{2} L_{2} \dot{\theta}_{2}^{2} \cos \theta_{2}+m_{3} \ddot{x}+\frac{m_{3} L \ddot{\theta} \sin \theta}{2}+\frac{m_{3} L \dot{\theta}^{2} \cos \theta}{2}-m_{3} L_{1} \ddot{\theta}_{1} \sin \theta_{1}$ $-m_{3} L_{1} \dot{\theta}_{1}^{2} \cos \theta_{1}-m_{3} L_{3}\left(\ddot{\theta}_{1}+\ddot{\theta}_{3}\right) \sin \left(\theta_{1}+\theta_{3}\right)-m_{3} L_{3}\left(\dot{\theta}_{1}+\dot{\theta}_{3}\right)^{2} \cos \left(\theta_{1}+\theta_{3}\right)+m_{4} \ddot{x}$ 


$$
\begin{aligned}
& -\frac{m_{4} L \ddot{\theta} \sin \theta}{2}-\frac{m_{4} L \dot{\theta}^{2} \cos \theta}{2}-m_{4} L_{2} \ddot{\theta}_{2} \sin \theta_{2}-m_{4} L_{2} \dot{\theta}_{2}^{2} \cos \theta_{2}-m_{4} L_{4}\left(\ddot{\theta}_{2}+\ddot{\theta}_{4}\right) \sin \left(\theta_{2}+\theta_{4}\right) \\
& -m_{4} L_{4}\left(\dot{\theta}_{2}+\dot{\theta}_{4}\right)^{2} \cos \left(\theta_{2}+\theta_{4}\right)+m_{5} \ddot{x}+\frac{m_{5} L \ddot{\theta} \sin \theta}{2}+\frac{m_{5} L \dot{\theta}^{2} \cos \theta}{2}-m_{5} L_{5} \ddot{\theta}_{5} \sin \theta_{5}-m_{5} L_{5} \dot{\theta}_{5}^{2} \cos \theta_{5}+m_{6} \ddot{x} \\
& -\frac{m_{6} L \ddot{\theta} \sin \theta}{2}-\frac{m_{6} L \dot{\theta}^{2} \cos \theta}{2}-m_{6} L_{6} \ddot{\theta}_{6} \sin \theta_{6}-m_{6} L_{6} \dot{\theta}_{6}^{2} \cos \theta_{6}+m_{7} \ddot{x}+\frac{m_{7} L \ddot{\theta} \sin \theta}{2}+\frac{m_{7} L \dot{\theta}^{2} \cos \theta}{2} \\
& -m_{7} L_{5} \ddot{\theta}_{5} \sin \theta_{5}-m_{7} L_{5} \dot{\theta}_{5}^{2} \cos \theta_{5}-m_{7} L_{7}\left(\ddot{\theta}_{5}+\ddot{\theta}_{7}\right) \sin \left(\theta_{5}+\theta_{7}\right)-m_{7} L_{7}\left(\dot{\theta}_{5}+\dot{\theta}_{7}\right)^{2} \cos \left(\theta_{5}+\theta_{7}\right) \\
& +m_{8} \ddot{x}-\frac{m_{8} L \ddot{\theta} \sin \theta}{2}-\frac{m_{8} L \dot{\theta}^{2} \cos \theta}{2}-m_{8} L_{6} \ddot{\theta}_{6} \sin \theta_{6}-m_{8} L_{6} \dot{\theta}_{6}^{2} \cos \theta_{6}-m_{8} L_{8}\left(\ddot{\theta}_{6}+\ddot{\theta}_{8}\right) \sin \left(\theta_{6}+\theta_{8}\right) \\
& -m_{8} L_{8}\left(\dot{\theta}_{6}+\dot{\theta}_{8}\right)^{2} \cos \left(\theta_{6}+\theta_{8}\right)+m_{n} \ddot{x}-m_{n} \mathrm{a} \ddot{\theta} \cos \theta+m_{n} \mathrm{a} \dot{\theta}^{2} \sin \theta-m_{n} \mathrm{~b}(\ddot{\alpha}+\ddot{\theta}) \sin (\alpha+\theta) \\
& -m_{n} \mathrm{~b}(\dot{\alpha}+\dot{\theta})^{2} \cos (\alpha+\theta)
\end{aligned}
$$

$$
\begin{aligned}
\sum \boldsymbol{F}_{\boldsymbol{y}} & =M \ddot{y}+m_{1} \ddot{y}-\frac{m_{1} L \ddot{\theta} \cos \theta}{2}+\frac{m_{1} L \dot{\theta}^{2} \sin \theta}{2}+m_{1} L_{1} \ddot{\theta}_{1} \cos \theta_{1}-m_{1} L_{1} \dot{\theta}_{1}^{2} \sin \theta_{1}+m_{2} \ddot{y}+\frac{m_{2} L \ddot{\theta} \cos \theta}{2}-\frac{m_{2} L \dot{\theta}^{2} \sin \theta}{2} \\
& +m_{2} L_{2} \ddot{\theta}_{2} \cos \theta_{2}-m_{2} L_{2} \dot{\theta}_{2}^{2} \sin \theta_{2}+m_{3} \ddot{y}-\frac{m_{3} L \ddot{\theta} \cos \theta}{2}+\frac{m_{3} L \dot{\theta}^{2} \sin \theta}{2}+m_{3} L_{1} \ddot{\theta}_{1} \cos \theta_{1} \quad-m_{3} L_{1} \dot{\theta}_{1}^{2} \sin \theta_{1} \\
& +m_{3} L_{3}\left(\ddot{\theta}_{1}+\ddot{\theta}_{3}\right) \cos \left(\theta_{1}+\theta_{3}\right)-m_{3} L_{3}\left(\dot{\theta}_{1}+\dot{\theta}_{3}\right)^{2} \sin \left(\theta_{1}+\theta_{3}\right)+m_{4} \ddot{y}+\frac{m_{4} L \ddot{\theta} \cos \theta}{2}-\frac{m_{4} L \dot{\theta}^{2} \sin \theta}{2} \\
& +m_{4} L_{2} \ddot{\theta}_{2} \cos \theta_{2}-m_{4} L_{2} \dot{\theta}_{2}^{2} \sin \theta_{2}+m_{4} L_{4}\left(\ddot{\theta}_{2}+\ddot{\theta}_{4}\right) \cos \left(\theta_{2}+\theta_{4}\right)-m_{4} L_{4}\left(\dot{\theta}_{2}+\dot{\theta}_{4}\right)^{2} \sin \left(\theta_{2}+\theta_{4}\right)+m_{5} \ddot{y} \\
& -\frac{m_{5} L \ddot{\theta} \cos \theta}{2}+\frac{m_{5} L \dot{\theta}^{2} \sin \theta}{2}+m_{5} L_{5} \ddot{\theta}_{5} \cos \theta_{5}-m_{5} L_{5} \dot{\theta}_{5}^{2} \sin \theta_{5}+m_{6} \ddot{y}+\frac{m_{6} L \ddot{\theta} \cos \theta}{2}-\frac{m_{6} L \dot{\theta}^{2} \sin \theta}{2} \\
& +m_{6} L_{6} \ddot{\theta}_{6} \cos \theta_{6}-m_{6} L_{6} \dot{\theta}_{6}^{2} \sin \theta_{6}+m_{7} \ddot{y}-\frac{m_{7} L \ddot{\theta} \cos \theta}{2}+\frac{m_{7} L \dot{\theta}^{2} \sin \theta}{2}+m_{7} L_{5} \ddot{\theta}_{5} \cos \theta_{5}-m_{7} L_{5} \dot{\theta}_{5}^{2} \sin \theta_{5} \\
& +m_{7} L_{7}\left(\ddot{\theta}_{5}+\ddot{\theta}_{7}\right) \cos \left(\theta_{5}+\theta_{7}\right)-m_{7} L_{7}\left(\dot{\theta}_{5}+\dot{\theta}_{7}\right)^{2} \sin \left(\theta_{5}+\theta_{7}\right)+m_{8} \ddot{y}+\frac{m_{8} L \ddot{\theta} \cos \theta}{2}-\frac{m_{8} L \dot{\theta}^{2} \sin \theta}{2} \\
& +m_{8} L_{6} \ddot{\theta}_{6} \cos \theta_{6}-m_{8} L_{6} \dot{\theta}_{6}^{2} \sin \theta_{6}+m_{8} L_{8}\left(\ddot{\theta}_{6}+\ddot{\theta}_{8}\right) \cos \left(\theta_{6}+\theta_{8}\right)-m_{8} L_{8}\left(\dot{\theta}_{6}+\dot{\theta}_{8}\right)^{2} \sin \left(\theta_{6}+\theta_{8}\right) \\
& +m_{n} \ddot{y}-m_{n} \mathrm{a} \ddot{\theta} \sin \theta-m_{n} \mathrm{a} \dot{\theta}^{2} \cos \theta+m_{n} \mathrm{~b}(\ddot{\alpha}+\ddot{\theta}) \cos (\alpha+\theta)-m_{n} \mathrm{~b}(\dot{\alpha}+\dot{\theta})^{2} \sin (\alpha+\theta)+M g \\
& +m_{1} g+m_{2} g+m_{3} g+m_{4} g+m_{5} g+m_{6} g+m_{7} g+m_{8} g+m_{n} g
\end{aligned}
$$

$$
\begin{aligned}
\boldsymbol{\tau} & =I \ddot{\theta}+\frac{m_{1} L^{2} \ddot{\theta}}{4}+\frac{m_{1} L \ddot{x} \sin \theta}{2}-\frac{m_{1} L \ddot{y} \cos \theta}{2}-\frac{m_{1} L L_{1} \dot{\theta}_{1} \cos \left(\theta-\theta_{1}\right)}{2}+\frac{m_{1} L L_{1} \dot{\theta}_{1}\left(\dot{\theta}-\dot{\theta}_{1}\right) \sin \left(\theta-\theta_{1}\right)}{2}+\frac{m_{2} L^{2} \ddot{\theta}}{4}-\frac{m_{2} L \ddot{x} \sin \theta}{2} \\
& +\frac{m_{2} L \ddot{y} \cos \theta}{2}+\frac{m_{2} L L_{2} \dot{\theta}_{2} \cos \left(\theta-\theta_{2}\right)}{2}-\frac{m_{2} L L_{2} \dot{\theta}_{2}\left(\dot{\theta}-\dot{\theta}_{2}\right) \sin \left(\theta-\theta_{2}\right)}{2}+\frac{m_{3} L^{2} \ddot{\theta}}{4}+\frac{m_{3} L \ddot{x} \sin \theta}{2}+\frac{m_{3} L L_{3} \dot{\theta}_{1}\left(\dot{\theta}-\dot{\theta}_{1}\right) \sin \left(\theta-\theta_{1}\right)}{2} \\
& -\frac{m_{3} L L_{3}\left(\ddot{\theta}_{1}+\ddot{\theta}_{3}\right) \cos \left(\theta-\theta_{1}-\theta_{3}\right)}{2}+\frac{m_{3} L L_{3}\left(\dot{\theta}_{1}+\dot{\theta}_{3}\right)\left(\dot{\theta}-\dot{\theta}_{1}-\dot{\theta}_{3}\right) \sin \left(\theta-\theta_{1}-\theta_{3}\right)}{2}+\frac{m_{4} L^{2} \ddot{\theta}}{4}-\frac{m_{4} L \ddot{x} \sin \theta}{2}+\frac{m_{4} L \ddot{y} \cos \theta}{2} \\
& +\frac{m_{4} L L_{2} \dot{\theta}_{2} \cos \left(\theta-\theta_{2}\right)}{2}-\frac{m_{4} L L_{2} \dot{\theta}_{2}\left(\dot{\theta}-\dot{\theta}_{2}\right) \sin \left(\theta-\theta_{2}\right)}{2}+\frac{m_{4} L L_{4}\left(\ddot{\theta}_{2}+\ddot{\theta}_{4}\right) \cos \left(\theta-\theta_{2}-\theta_{4}\right)}{2}-\frac{m_{4} L L_{4}\left(\dot{\theta}_{2}+\dot{\theta}_{4}\right)\left(\dot{\theta}-\dot{\theta}_{2}-\dot{\theta}_{4}\right) \sin \left(\theta-\theta_{2}-\theta_{4}\right)}{2} \\
& +\frac{m_{5} L^{2} \ddot{\theta}}{4}+\frac{m_{5} L \ddot{x} \sin \theta}{2}-\frac{m_{5} L \ddot{y} \cos \theta}{2}-\frac{m_{5} L L_{5} \dot{\theta}_{5} \cos \left(\theta-\theta_{5}\right)}{2}+\frac{m_{5} L L_{5} \dot{\theta}_{5}\left(\dot{\theta}-\dot{\theta}_{5}\right) \sin \left(\theta-\theta_{5}\right)}{2}+\frac{m_{6} L^{2} \ddot{\theta}}{4}-\frac{m_{6} L \ddot{x} \sin \theta}{2} \\
& +\frac{m_{6} L \ddot{y} \cos \theta}{2}+\frac{m_{6} L L_{6} \ddot{\theta}_{6} \cos \left(\theta-\theta_{6}\right)}{2}-\frac{m_{6} L L_{6} \dot{\theta}_{6}\left(\dot{\theta}-\dot{\theta}_{6}\right) \sin \left(\theta-\theta_{6}\right)}{2}+\frac{m_{7} L^{2} \ddot{\theta}}{4}+\frac{m_{7} L \ddot{x} \sin \theta}{2}-\frac{m_{7} L \ddot{y} \cos \theta}{2}-\frac{m_{7} L L_{5} \dot{\theta}_{5} \cos \left(\theta-\theta_{5}\right)}{2} \\
& +\frac{m_{7} L L_{7} \dot{\theta}_{5}\left(\dot{\theta}-\dot{\theta}_{5}\right) \sin \left(\theta-\theta_{5}\right)}{2}-\frac{m_{7} L L_{7}\left(\ddot{\theta}_{5}+\ddot{\theta}_{7}\right) \cos \left(\theta-\theta_{5}-\theta_{7}\right)}{2}+\frac{m_{7} L L_{7}\left(\dot{\theta}_{5}+\dot{\theta}_{7}\right)\left(\dot{\theta}-\dot{\theta}_{5}-\dot{\theta}_{7}\right) \sin \left(\theta-\theta_{5}-\theta_{7}\right)}{2}+\frac{m_{8} L^{2} \ddot{\theta}}{4}-\frac{m_{8} L \ddot{x} \sin \theta}{2} \\
& +\frac{m_{8} L \ddot{y} \cos \theta}{2}+\frac{m_{8} L L_{6} \dot{\theta}_{6} \cos \left(\theta-\theta_{6}\right)}{2}-\frac{m_{8} L L_{6} \dot{\theta}_{6}\left(\dot{\theta}-\dot{\theta}_{6}\right) \sin \left(\theta-\theta_{6}\right)}{2}+\frac{m_{8} L L_{8}\left(\ddot{\theta}_{6}+\ddot{\theta}_{8}\right) \cos \left(\theta-\theta_{6}-\theta_{8}\right)}{2}-\frac{m_{1} L L_{1} \dot{\theta} \dot{\theta}_{1} \sin \left(\theta-\theta_{1}\right)}{2}
\end{aligned}
$$




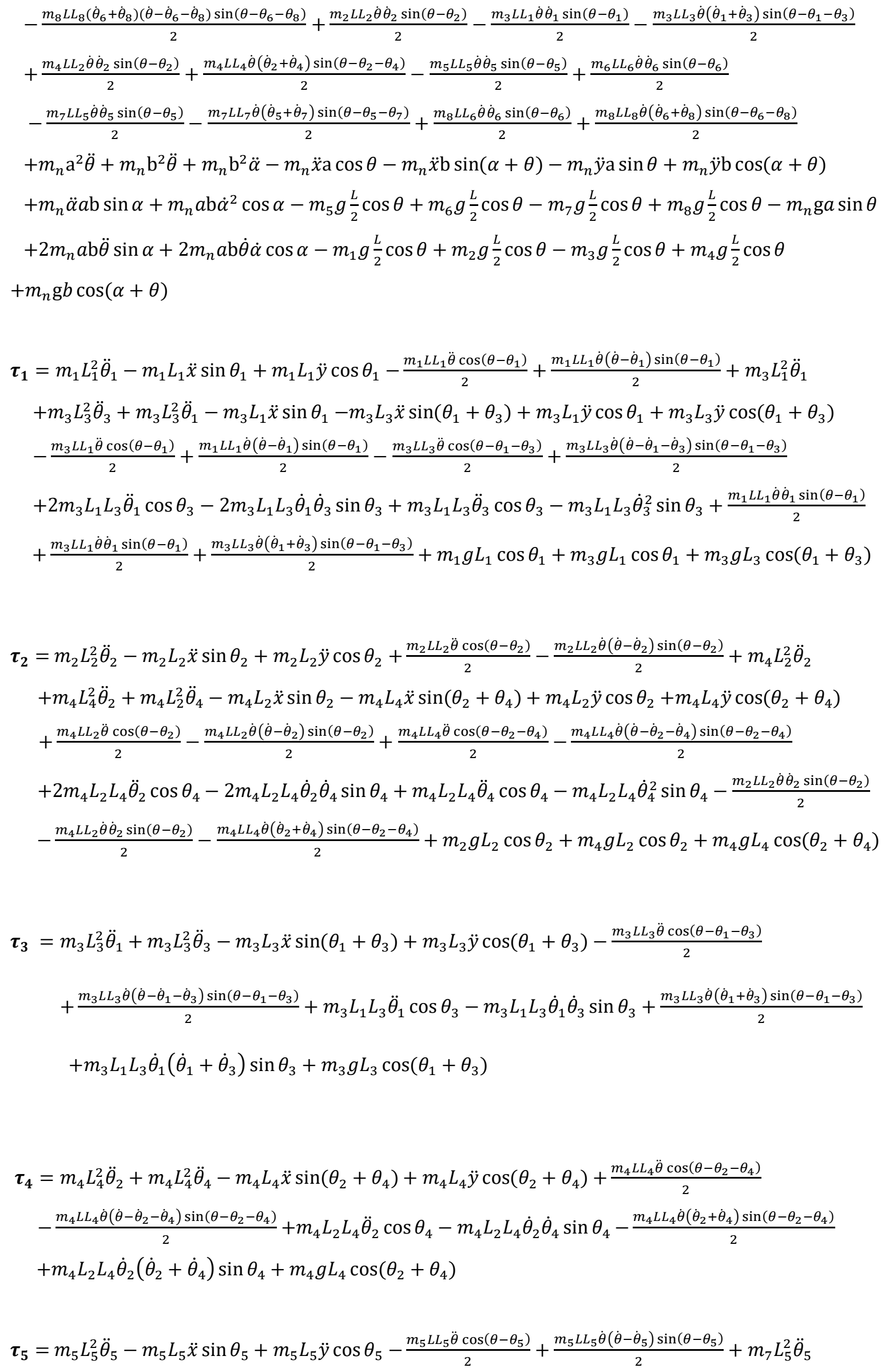




$$
\begin{aligned}
& +m_{7} L_{7}^{2} \ddot{\theta}_{7}+m_{7} L_{7}^{2} \ddot{\theta}_{5}-m_{7} L_{5} \ddot{x} \sin \theta_{5}-m_{7} L_{7} \ddot{x} \sin \left(\theta_{5}+\theta_{7}\right)+m_{7} L_{5} \ddot{y} \cos \theta_{5}+m_{7} L_{7} \ddot{y} \cos \left(\theta_{5}+\theta_{7}\right) \\
& -\frac{m_{7} L L_{5} \ddot{\theta} \cos \left(\theta-\theta_{5}\right)}{2}+\frac{m_{5} L L_{5} \dot{\theta}\left(\dot{\theta}-\dot{\theta}_{5}\right) \sin \left(\theta-\theta_{5}\right)}{2}-\frac{m_{7} L L_{7} \ddot{\theta} \cos \left(\theta-\theta_{5}-\theta_{7}\right)}{2}+\frac{m_{7} L L_{7} \dot{\theta}\left(\dot{\theta}-\dot{\theta}_{5}-\dot{\theta}_{7}\right) \sin \left(\theta-\theta_{5}-\theta_{7}\right)}{2} \\
& +2 m_{7} L_{5} L_{7} \ddot{\theta}_{5} \cos \theta_{7}-2 m_{7} L_{5} L_{7} \dot{\theta}_{5} \dot{\theta}_{7} \sin \theta_{7}+m_{7} L_{5} L_{7} \ddot{\theta}_{7} \cos \theta_{7}-m_{7} L_{5} L_{7} \dot{\theta}_{7}^{2} \sin \theta_{7}+\frac{m_{5} L L_{5} \dot{\theta} \dot{\theta}_{5} \sin \left(\theta-\theta_{5}\right)}{2} \\
& +\frac{m_{7} L L_{5} \dot{\theta} \dot{\theta}_{5} \sin \left(\theta-\theta_{5}\right)}{2}+\frac{m_{7} L L_{7} \dot{\theta}\left(\dot{\theta}_{5}+\dot{\theta}_{7}\right) \sin \left(\theta-\theta_{5}-\theta_{7}\right)}{2}+m_{5} g L_{5} \cos \theta_{5}+m_{7} g L_{5} \cos \theta_{5}+m_{7} g L_{7} \cos \left(\theta_{5}+\theta_{7}\right) \\
& \begin{aligned}
\boldsymbol{\tau}_{6} & =m_{6} L_{6}^{2} \ddot{\theta}_{6}-m_{6} L_{6} \ddot{x} \sin \theta_{6}+m_{6} L_{6} \ddot{y} \cos \theta_{6}+\frac{m_{6} L L_{6} \ddot{\theta} \cos \left(\theta-\theta_{6}\right)}{2}-\frac{m_{6} L L_{6} \dot{\theta}\left(\dot{\theta}-\dot{\theta}_{6}\right) \sin \left(\theta-\theta_{6}\right)}{2}+m_{8} L_{6}^{2} \ddot{\theta}_{6} \\
& +m_{8} L_{8}^{2} \ddot{\theta}_{6}+m_{8} L_{6}^{2} \ddot{\theta}_{8}-m_{8} L_{6} \ddot{x} \sin \theta_{6}-m_{8} L_{8} \ddot{x} \sin \left(\theta_{6}+\theta_{8}\right)+m_{8} L_{6} \ddot{y} \cos \theta_{6}+m_{8} L_{8} \ddot{y} \cos \left(\theta_{6}+\theta_{8}\right) \\
& -\frac{m_{8} L L_{6} \dot{\theta}\left(\dot{\theta}-\dot{\theta}_{6}\right) \sin \left(\theta-\theta_{6}\right)}{2}+\frac{m_{8} L L_{8} \ddot{\theta} \cos \left(\theta-\theta_{6}-\theta_{8}\right)}{2}+2 m_{8} L_{6} L_{8} \ddot{\theta}_{6} \cos \theta_{8}-\frac{m_{8} L L_{8} \dot{\theta}\left(\dot{\theta}-\dot{\theta}_{6}-\dot{\theta}_{8}\right) \sin \left(\theta-\theta_{6}-\theta_{8}\right)}{2} \\
& -2 m_{8} L_{6} L_{8} \dot{\theta}_{6} \dot{\theta}_{8} \sin \theta_{8}+m_{8} L_{6} L_{8} \ddot{\theta}_{8} \cos \theta_{8}-m_{8} L_{6} L_{8} \dot{\theta}_{8}^{2} \sin \theta_{8}-\frac{m_{6} L L_{6} \dot{\theta} \dot{\theta}_{6} \sin \left(\theta-\theta_{6}\right)}{2}-\frac{m_{8} L L_{6} \dot{\theta} \dot{\theta}_{6} \sin \left(\theta-\theta_{6}\right)}{2} \\
& -\frac{m_{8} L L_{8} \dot{\theta}\left(\dot{\theta}_{6}+\dot{\theta}_{8}\right) \sin \left(\theta-\theta_{6}-\theta_{8}\right)}{2}+m_{6} g L_{6} \cos \theta_{6}+m_{8} g L_{6} \cos \theta_{6}+m_{8} g L_{8} \cos \left(\theta_{6}+\theta_{8}\right)
\end{aligned} \\
& \boldsymbol{\tau}_{7}=m_{7} L_{7}^{2} \ddot{\theta}_{5}+m_{7} L_{7}^{2} \ddot{\theta}_{7}-m_{7} L_{7} \ddot{x} \sin \left(\theta_{5}+\theta_{7}\right)+m_{7} L_{7} \ddot{y} \cos \left(\theta_{5}+\theta_{7}\right)-\frac{m_{7} L L_{7} \ddot{\theta} \cos \left(\theta-\theta_{5}-\theta_{7}\right)}{2} \\
& +\frac{m_{7} L L_{7} \dot{\theta}\left(\dot{\theta}-\dot{\theta}_{5}-\dot{\theta}_{7}\right) \sin \left(\theta-\theta_{5}-\theta_{7}\right)}{2}+m_{7} L_{5} L_{7} \ddot{\theta}_{5} \cos \theta_{7}-m_{7} L_{5} L_{7} \dot{\theta}_{5} \dot{\theta}_{7} \sin \theta_{7}+\frac{m_{7} L L_{7} \dot{\theta}\left(\dot{\theta}_{5}+\dot{\theta}_{7}\right) \sin \left(\theta-\theta_{5}-\theta_{7}\right)}{2} \\
& +m_{7} L_{5} L_{7} \dot{\theta}_{5}\left(\dot{\theta}_{5}+\dot{\theta}_{7}\right) \sin \theta_{7}+m_{7} g L_{7} \cos \left(\theta_{5}+\theta_{7}\right) \\
& \boldsymbol{\tau}_{\mathbf{8}}=m_{8} L_{8}^{2} \ddot{\theta}_{8}+m_{8} L_{8}^{2} \ddot{\theta}_{8}-m_{8} L_{8} \ddot{x} \sin \left(\theta_{6}+\theta_{8}\right)+m_{8} L_{8} \ddot{y} \cos \left(\theta_{6}+\theta_{8}\right)+\frac{m_{8} L L_{8} \ddot{\theta} \cos \left(\theta-\theta_{6}-\theta_{8}\right)}{2} \\
& -\frac{m_{8} L L_{8} \dot{\theta}\left(\dot{\theta}-\dot{\theta}_{6}-\dot{\theta}_{8}\right) \sin \left(\theta-\theta_{6}-\theta_{8}\right)}{2}+m_{8} L_{6} L_{8} \ddot{\theta}_{6} \cos \theta_{8}-m_{8} L_{6} L_{8} \dot{\theta}_{6} \dot{\theta}_{8} \sin \theta_{8}-\frac{m_{8} L L_{8} \dot{\theta}_{(}\left(\dot{\theta}_{6}+\dot{\theta}_{8}\right) \sin \left(\theta-\theta_{6}-\theta_{8}\right)}{2} \\
& +m_{8} L_{6} L_{8} \dot{\theta}_{6}\left(\dot{\theta}_{6}+\dot{\theta}_{8}\right) \sin \theta_{8}+m_{8} g L_{8} \cos \left(\theta_{6}+\theta_{8}\right)
\end{aligned}
$$

3.

$$
\begin{aligned}
& {\left[J_{R_{\text {omuz }}}\right]=\left[\begin{array}{rrrrrrrrrrr}
1 & 0 & (-L / 2) \cdot \sin \theta & 0 & 0 & 0 & 0 & 0 & 0 & 0 & 0 \\
0 & 1 & (L / 2) \cdot \cos \theta & 0 & 0 & 0 & 0 & 0 & 0 & 0 & 0
\end{array}\right]} \\
& {\left[J_{\text {omuz }}\right]=\left[\begin{array}{ccccccccccc}
0 & 0 & (L / 2) \cdot \sin \theta & 0 & 0 & 0 & 0 & 0 & 0 & 0 & 0 \\
1 & 1 & (-L / 2) \cdot \cos \theta & 0 & 0 & 0 & 0 & 0 & 0 & 0 & 0
\end{array}\right]}
\end{aligned}
$$$$
\left[J_{1}\right]=\left[\begin{array}{rrrrrrrrrrr}
1 & 0 & (L / 2) \cdot \sin \theta-L_{1} \cdot \sin \theta_{1} & -L_{1} \cdot \sin \theta_{1} & 0 & 0 & 0 & 0 & 0 & 0 & 0 \\
0 & 1 & (-L / 2) \cdot \cos \theta+L_{1} \cdot \cos \theta_{1} & L_{1} \cdot \cos \theta_{1} & 0 & 0 & 0 & 0 & 0 & 0 & 0
\end{array}\right]
$$$$
\left[J_{2}\right]=\left[\begin{array}{rrrrrrrrrrr}
1 & 0 & (-L / 2) \cdot \sin \theta-L_{2} \cdot \sin \theta_{2} & 0 & -L_{2} \cdot \sin \theta_{2} & 0 & 0 & 0 & 0 & 0 & 0 \\
0 & 1 & (L / 2) \cdot \cos \theta+L_{2} \cdot \cos \theta_{2} & 0 & L_{2} \cdot \cos \theta_{2} & 0 & 0 & 0 & 0 & 0 & 0
\end{array}\right]
$$

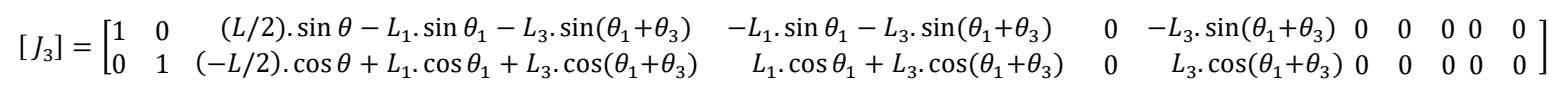




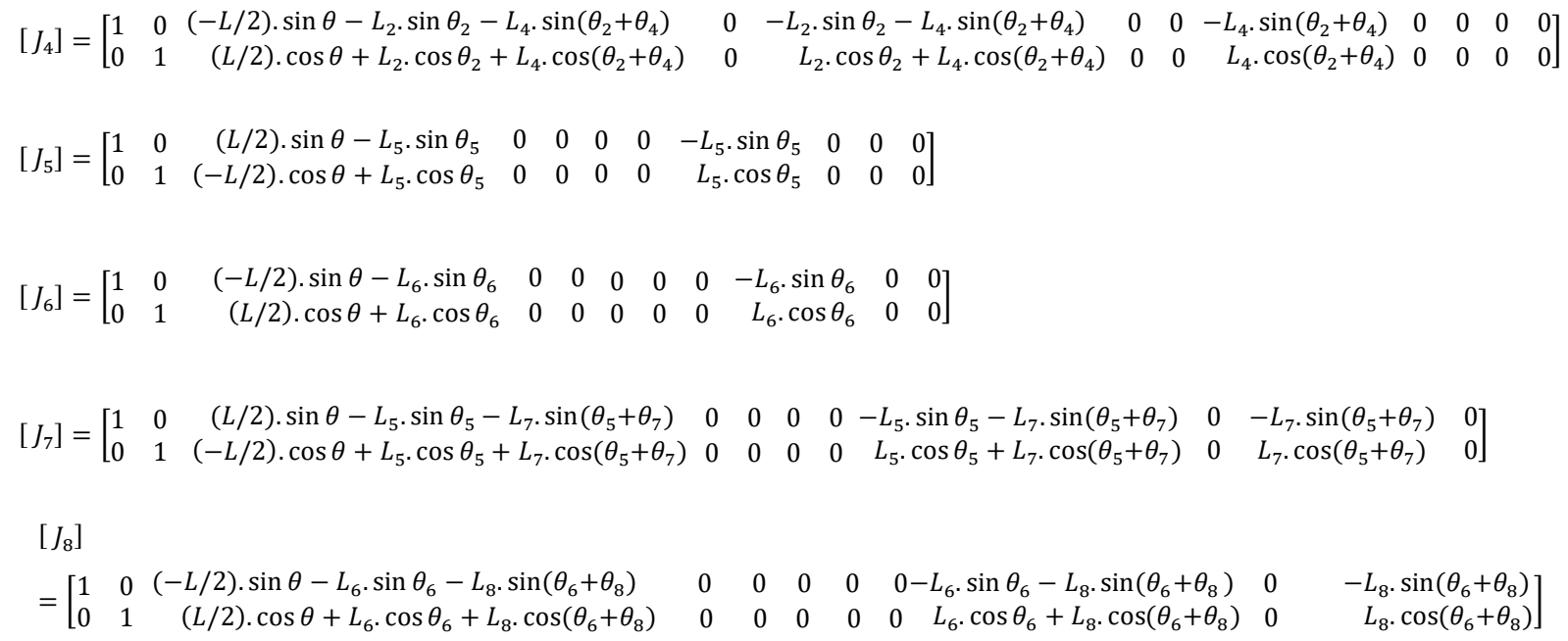

